\title{
Investigation of Magnetic Dead Layer on Iron Silicide Surfaces*
}

\author{
Mohammad Tawheed Kibria, ${ }^{\dagger}$ Tatsushi Nomitsu, Takeshi Nakagawa, ${ }^{\ddagger}$ and Seigi Mizuno \\ Department of Molecular and Material Sciences, Interdisciplinary Graduate School of Engineering Sciences, \\ Kyushu University, 6-1 Kasuga Koen, Kasuga, Fukuoka 816-8580, Japan \\ (Received 27 December 2017; Accepted 9 March 2018; Published 26 April 2018)
}

\begin{abstract}
We report the magnetic dead layer at the interfaces between $\mathrm{Fe}$ and $\mathrm{Si}(111)$ surfaces. We prepared two ultrathin iron-silicides, $\mathrm{c}(8 \times 4)$ prepared at $900 \mathrm{~K}$ and $\mathrm{p}(2 \times 2)$ at $700 \mathrm{~K}$, and clean reconstructed $7 \times 7$ surface for the silicon templates. By using magneto-optical Kerr effect, we found that at room temperature the ferromagnetic order appears at 5.5 ML, 8.2 ML, and 10.9 ML for Fe/c $(8 \times 4)$ silicide, Fe/p $(2 \times 2)$ silicide, and $\mathrm{Fe} / \mathrm{Si}(111)-7 \times 7$ surface, respectively. From the thickness dependent magnetization measurements, we decided the magnetic dead layer for $\mathrm{Fe} / \mathrm{c}(8 \times 4)$ silicide, $\mathrm{Fe} / \mathrm{p}(2 \times 2)$ silicide, and $\mathrm{Fe} / \mathrm{Si}(111)-7 \times 7$ to be $3.0 \mathrm{ML}, 5.0 \mathrm{ML}$, and $7.5 \mathrm{ML}$, respectively. Our results indicate that the $\mathrm{c}(8 \times 4)$ iron silicide surface prevents further silicide formation more effectively than the $\mathrm{p}(2 \times 2)$ silicide and $\mathrm{Si}(111)-7 \times 7$ surface. [DOI: $10.1380 /$ ejssnt.2018.101]
\end{abstract}

Keywords: Low energy electron diffraction (LEED); Magnetic Measurements; Iron; Silicon; Magnetic phenomena

\section{INTRODUCTION}

The ferromagnetic behavior of ultrathin iron films on Si substrates has been extensively studied for the magnetic device's applications [1-13]. Substantial intermixing, however, occurs at the $\mathrm{Fe} / \mathrm{Si}$ interface even at room temperature (RT) due to high reactivity of Si. The intermixing is accountable for no magnetic moment at the interfaces between $\mathrm{Fe}$ and $\mathrm{Si}$, which is called a magnetic dead layer (MDL) [8]. MDL has been a crucial concern since MDL would affect the spin transport [14].

The formation of the intermixing critically depends on film thickness, structure, and stoichiometry of the FeSi interface [15-23]. To avoid the strong intermixing of $\mathrm{Si}$ atoms with ferromagnetic metals and to maintain the large magnetic moments of the ferromagnetic metal atoms, several methods have been proposed: the insertion of thin buffer layers such as metallic [24-26], semiconducting [11], insulating [4] materials, passivation of the $\mathrm{Si}$ surface with other materials to form inert interfaces [29]. The passivation of $\mathrm{Si}(111)$ surface with nitrogen, forming a stable nitride, is successful, resulting in the absence of the magnetic dead layer [29]. On the Si nitride surface, however, the subsequently grown Fe forms nanoparticles instead of films, and is superparamagnetic for low coverages $(\sim 2 \mathrm{ML})$. For the epitaxial growth of Fe, an ironsilicide buffer layer would be suitable since it was reported that $\mathrm{Fe}(111)$ orientated films grow on the iron silicide surface [7].

A lot of research has been devoted to the study of the interfacial structure and magnetism of Fe films such as $\beta-\mathrm{FeSi}_{2}$ and $\varepsilon$-FeSi on Si substrates $[27,28]$. The investigation of the interfacial MDL for ultrathin Fe films on iron silicides and $\mathrm{Si}(111)-7 \times 7$ surfaces plays an important role for the heterojunction. As previously reported [30, 31], the high-quality epitaxial ultrathin iron silicides can be obtained on $\mathrm{Si}(111)$. These iron silicides are thermally stable, and could be better candidates for the suppression of the interfacial MDL of Fe-Si alloy.

In this study, the ultrathin iron silicides were used as templetes for the reduction of MDL. Magnetization curves

\footnotetext{
* This paper was presented at the 8th International Symposium on Surface Science, Tsukuba International Congress Center, Tsukuba, Japan, October 22-26, 2017.

† Corresponding author: kibria.tawheed@kyudai.jp

¥ Corresponding author: naka@kyudai.jp
}

at the interface of the additional Fe films on iron silicide phases, $\mathrm{c}(8 \times 4)$ and $\mathrm{p}(2 \times 2)$, was measured by magnetooptic Kerr effect (MOKE), and the interfacial MDL on iron silicide phases, $\mathrm{c}(8 \times 4)$ and $\mathrm{p}(2 \times 2)$ was evaluated. The results of MDL for the silicide templates were compared with that for the clean $\operatorname{Si}(111)-7 \times 7$ surface.

\section{EXPERIMENTAL}

The experiments were conducted in a multi-chamber UHV system with a base pressure of $2 \times 10^{-8} \mathrm{~Pa}$, allowing in situ transfer between the chambers for low energy electron diffraction (LEED) and MOKE measurements. A Si(111) substrate was cut from a commercial Si wafer. The Si substrate was washed by ultrasonic cleaning with ethanol, and then fixed on a nonmagnetic sample holder. The substrate was heated by electron bombardment, degassed at $800 \mathrm{~K}$ for $12 \mathrm{~h}$, and repeatedly flashed at approximately $1400 \mathrm{~K}$. After this procedure, a clean reconstructed $\mathrm{Si}(111)-7 \times 7$ surface was confirmed by LEED as shown in Fig. 1(c). Iron silicide layers were grown with Fe deposited onto a $\mathrm{Si}(111)-7 \times 7$ surface at RT and subsequent anneal at 700-900 K for $10 \mathrm{~min}$. Fe was evaporated from an electron beam evaporator. The pressure during the $\mathrm{Fe}$ deposition was below $4 \times 10^{-8} \mathrm{~Pa}$. The Fe deposition rate $(\sim 1 \mathrm{ML} / \mathrm{min})$ and Fe coverage $\left(\theta_{\mathrm{Fe}}\right)$ were monitored by a quartz crystal microbalance. Here $1 \mathrm{ML}$ is defined as $7.8 \times 10^{14}$ atoms $/ \mathrm{cm}^{2}$, which corresponds to the surface atomic density of $\mathrm{Si}(111)$. This atomic density is close to that of bcc Fe(111), $7.1 \times 10^{14}$ atoms $/ \mathrm{cm}^{2}$, which is known to grow on $\mathrm{Si}(111)$ for thicker Fe coverages $(>10 \mathrm{ML})[7]$.

The magnetic properties were measured by in situ MOKE with an electromagnet applying up to 1000 Oe. For the MOKE measurements, a 635-nm laser diode was used as a light source, and the light incident angle was set at $45^{\circ}$ on the sample.

\section{RESULTS}

\section{A. Iron silicide formation}

Ultrathin iron silicides were prepared on $\mathrm{Si}(111)$ surface in the same way as previous reports [30, 31]. 1.7 ML 

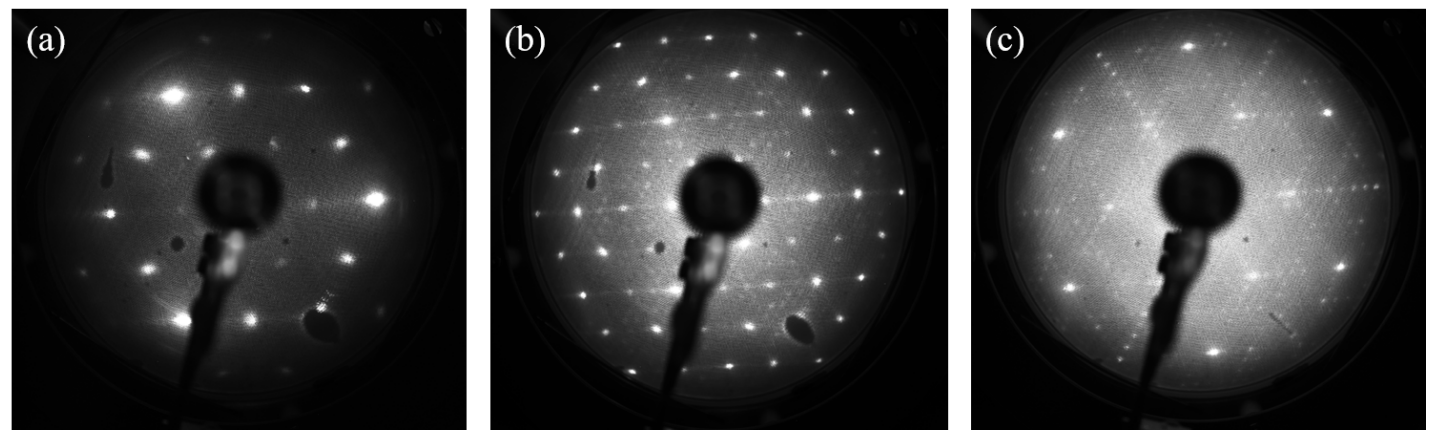

FIG. 1. LEED patterns for three templates; (a) $\mathrm{p}(2 \times 2)$ iron silicide (the electron energy at $51 \mathrm{eV})$, (b) $\mathrm{c}(8 \times 4)$ iron silicide (the electron energy at $99 \mathrm{eV}$ ), and (c) clean $\mathrm{Si}(111)-7 \times 7$ surface (the electron energy at $110 \mathrm{eV}$ ).
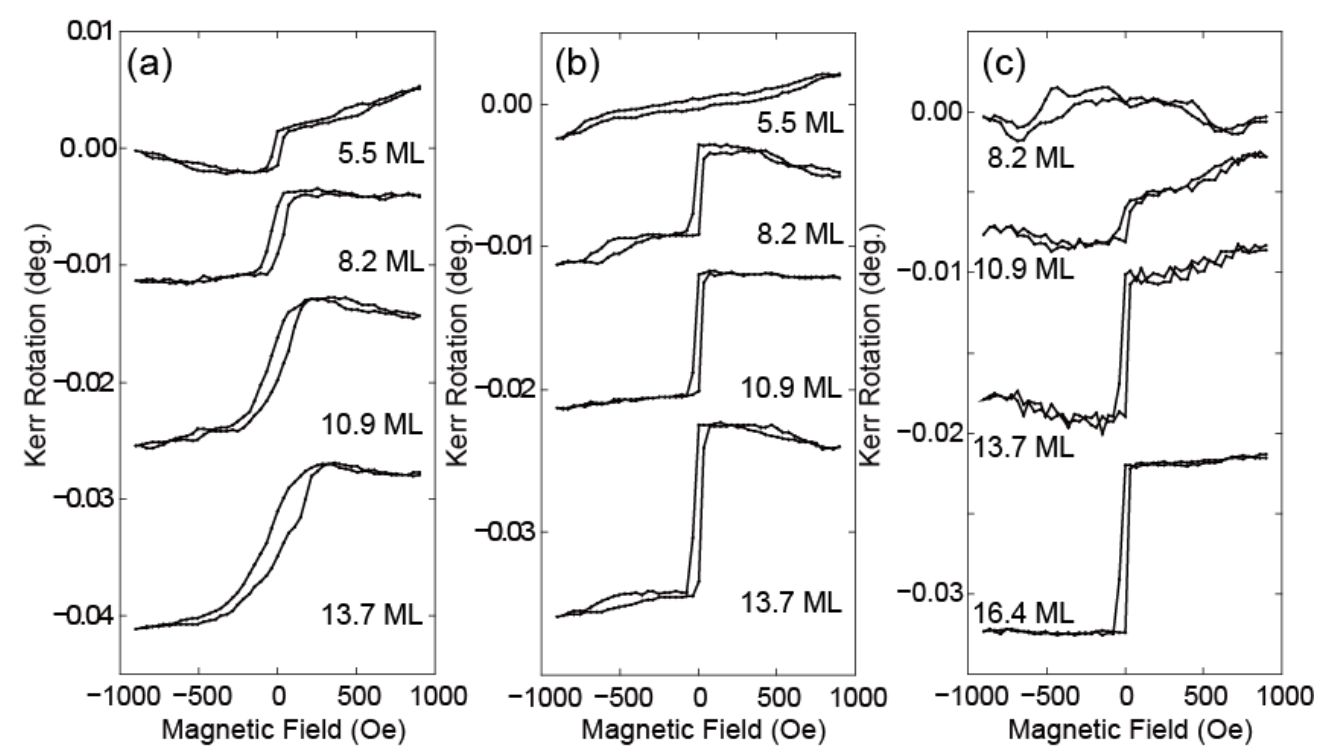

FIG. 2. Magnetization loops by the longitudinal MOKE as a function of Fe coverage on (a) c( $8 \times 4)$ silicide, (b) p $(2 \times 2)$ silicide, and (c) $\mathrm{Si}(111)-7 \times 7$ surface. Indicated in the plots are the Fe coverages.

Fe was deposited on $\mathrm{Si}(111)$ at RT, which was subsequently annealed. Depending on the annealing temperature, two different iron silicides were formed as observed by LEED [Fig. 1(a, b)]. Figure $1(\mathrm{a})$ shows a $\mathrm{p}(2 \times 2)$ structure obtained at the annealing temperature of 700 $\mathrm{K}$. With increasing the annealing temperature upto 900 $\mathrm{K}$, the $\mathrm{p}(2 \times 2)$ structure transformed to the $\mathrm{c}(8 \times 4)$ silicide [Fig. 1(b)], which is in good agreement with the previous reports $[8,30,31]$. With further increase of the annealing temperature, the $c(8 \times 4)$ structure gradually changed to the $(7 \times 7)$ structure, which indicates the recovery of the clean $\mathrm{Si}(111)$ surface by the diffusion of $\mathrm{Fe}$ into deeper bulk. The $\mathrm{c}(8 \times 4)$ iron silicide phase did not appear at the higher coverage of $\theta_{\mathrm{Fe}} \simeq 4 \mathrm{ML}$, while the $\mathrm{p}(2 \times 2)$ silicide phase appeared at higher Fe coverages $\left(\theta_{\mathrm{Fe}}>4 \mathrm{ML}\right)$ [8]. These iron silicides were not ferromagnetic and we used them as templates for the growth of additional $\mathrm{Fe}$ thin films.

\section{B. Magnetic properties}

The magnetic properties of the ultrathin $\mathrm{Fe}$ films upto $\sim 30 \mathrm{ML}$ on the iron silicides $[\mathrm{p}(2 \times 2)$ and $\mathrm{c}(8 \times 4)]$ and $\operatorname{Si}(111)-7 \times 7$ surface were investigated at RT using MOKE. The Fe coverage shown does not include the Fe amount used for the silicide formation. The longitudinal geometry, i.e., the magnetic field applied within the plane of the films, was employed to measure magnetization hysteresis curves throughout the whole Fe thickness range. Typical magnetization loops with different Fe coverages at RT for two iron silicide surfaces $[\mathrm{c}(8 \times 4)$ and $\mathrm{p}(2 \times 2)]$ and $\mathrm{Si}(111)-7 \times 7$ surface are plotted in Figs. 2(a), 2(b), and 2(c), respectively. Below the Fe coverage of $5 \mathrm{ML}$, there was no indication of a ferromagnetic (FM) order on the iron silicides and $\mathrm{Si}(111)-7 \times 7$ surfaces. With increasing the Fe coverage, FM was observed for Fe/c $(8 \times 4)$ iron silicide at $\theta_{\mathrm{Fe}}=5.5 \mathrm{ML}$. For $\mathrm{Fe} / \mathrm{p}(2 \times 2)$ iron silicide $\mathrm{FM}$ was observed at $\theta_{\mathrm{Fe}}=8.2 \mathrm{ML}$, although the magnetization loop at $\theta_{\mathrm{Fe}}=5.5 \mathrm{ML}$ shows monotonic increase without distinct hysteresis, indicating that the $5.5 \mathrm{ML}$ film is on the verge of FM. For Fe/7×7-Si(111), FM appeared at $\theta_{\mathrm{Fe}}=10.9 \mathrm{ML}$.

To identify the MDL on the iron silicide and $\mathrm{Si}(111)$ $7 \times 7$ surface, we measured Fe thickness dependence of Kerr rotation. The Kerr rotation is approximately proportional to the total magnetization of the sample when the thickness of the sample is enough thin compared with 


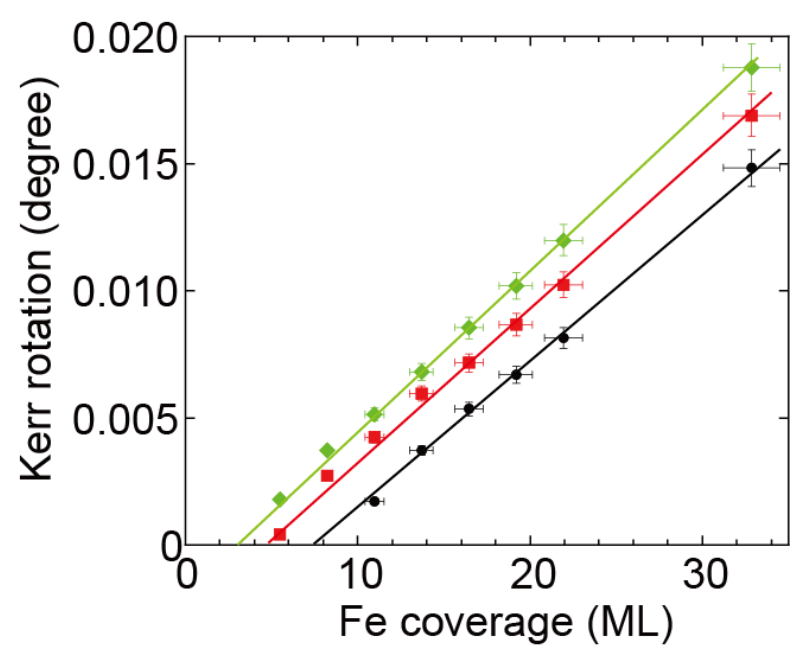

FIG. 3. Kerr rotation as a function of Fe coverage. Black, red, and green dots indicate Kerr rotation of Fe on $\mathrm{Si}(111)$ $7 \times 7, \mathrm{p}(2 \times 2)$, and $\mathrm{c}(8 \times 4)$, respectively. The straight lines are the fits for the Kerr rotation (see text), and the intersections of the Fe coverage at the zero Kerr rotation indicate the magnetic dead layers.

the light penetration depth [32]. Figure 3 shows the Kerr rotations as a function of Fe coverages on the iron silicides and $\operatorname{Si}(111)-7 \times 7$. The slopes for all three samples are very similar $\left(6.2 \times 10^{-4} \mathrm{deg} / \mathrm{ML}\right)$, indicating that the films with ferromagnetic order have similar electronic structures. The Fe films grown on the ordered silicides, $\mathrm{p}(2 \times 2)$ and $\mathrm{c}(4 \times 8)$ phases, and $\mathrm{Si}(111)-7 \times 7$ show epitaxial bcc structures [7, 29], with a preferential (111) orientation. The similar slope of the Kerr rotations for the three samples is in good agreement with these structural results.

Thickness dependence of the Kerr rotations shows that the Fe film on $\mathrm{c}(8 \times 4)$ silicide has larger Kerr rotations than those for the $\mathrm{p}(2 \times 2)$ silicide and $\mathrm{Si}(111)-7 \times 7$ surface. With increasing the Fe coverage, the Kerr rotation linearly increases. If the entire Fe film is ferromagnetic at the higher coverage, the intersection at zero Kerr rotation should correspond to zero Fe thickness [32, 33]. However this is not the case for the silicides and $\mathrm{Si}(111)$ templates, and the intersections show the magnetic dead layer. On $\mathrm{c}(8 \times 4)$ silicide, the extrapolated Kerr rotation is zero at 3 $\mathrm{ML}$, indicating that the MDL for $\mathrm{c}(8 \times 4)$ is $3 \mathrm{ML}$. In the same way, the Kerr rotation as a function of the Fe coverage indicates that the MDL is $5 \mathrm{ML}$ for $\mathrm{p}(2 \times 2)$ silicide and 7.5 ML for $\mathrm{Si}(111)-7 \times 7$ surface.

\section{DISCUSSION}

We were not able to obtain any ferromagnetic order at RT below $5 \mathrm{ML}$ of Fe coverage on all the samples studied, $\mathrm{c}(8 \times 4)$ silicide, $\mathrm{p}(2 \times 2)$ silicide, and $\mathrm{Si}(111)-7 \times 7$ substrate. This is due to the intermixing of Fe and Si, leading to the formation of thin non-magnetic Fe-Si interfacial phases [2]. On $\mathrm{Si}(111)-7 \times 7$ with Fe deposition at RT, the onset of ferromagnetism has been reported to be between 4.7-6 ML [2, 7, 29], which is slightly thinner than our result, 7.5 ML. This slight difference may come from the deposition conditions such as deposition rate, temperature since they affect the diffusion and morphology [35].

The MDL for the iron silicide templates is thinner than that for the clean $\mathrm{Si}(111)-7 \times 7$. The silicide substrates can prevent the diffusion of $\mathrm{Fe}$ at the interfaces more effectively. Although the same amount of Fe is deposited for the preparation of the silicide templates, the MDL for the $\mathrm{c}(8 \times 4)$ template is thinner than that for the $\mathrm{p}(2 \times 2)$. The difference of the MLD is attributed to the preparation temperatures and possible induced ferromagnetism at the interface with additional Fe deposition.

The interface Fe silicide can become ferromagnetic [28] due to the further silicide formation and the exchange interaction with the additional Fe films, which gradually proceeds as the Fe thickness increases. This would in part contribute to the thinner MDL for the $\mathrm{p}(2 \times 2)$ and $\mathrm{c}(8 \times 4)$ templates. Yet, the $\mathrm{c}(8 \times 4)$ template acts as more effective protective layer than $\mathrm{p}(2 \times 2)$, regardless of the ferromagnetic contribution at the interface.

The ultra-thin $\mathrm{c}(8 \times 4)$ and $\mathrm{p}(2 \times 2)$ Fe silicide phases are reported to have c-FeSi structure, which is restricted at the interface, not stable in the bulk phase [34]. Our results indicate that the $c(8 \times 4)$ Fe silicide surface prevents further silicide formation. The $\mathrm{c}(8 \times 4)$ surface has a higher disintegration temperature than $\mathrm{p}(2 \times 2)[30,31$. The thermal stability of the $\mathrm{c}(4 \times 8)$ structure is noticeable even when compared with thick Fe silicide (4 ML) with a $\mathrm{p}(2 \times 2)$ structure [30]. This thermally stable interface would sustain after additional Fe deposition.

\section{CONCLUSION}

To summarise, we have investigated the interfacial magnetism and a magnetic dead layer of ultrathin Fe film/iron silicides and $\mathrm{Si}(111)-7 \times 7$ interface at RT by MOKE. The MOKE result shows that $5.5 \mathrm{ML} \mathrm{Fe} / \mathrm{c}(8 \times 4)$ silicide, 8.2 $\mathrm{ML} \mathrm{Fe} / \mathrm{p}(2 \times 2)$ silicide, and $10 \mathrm{ML} \mathrm{Fe} / \mathrm{Si}(111)-7 \times 7$ interface set in ferromagnetical order at RT, respectively. This different behaviour of the Fe film on the iron silicides and $\operatorname{Si}(111)-7 \times 7$ surfaces is due to the interfacial intermixing between $\mathrm{Fe}$ and $\mathrm{Si}$ atoms. The thickness dependent study of the Kerr rotations shows that the MDL for $\mathrm{Fe} / \mathrm{c}(8 \times 4)$ silicide is $3.0 \mathrm{ML}, \mathrm{Fe} / \mathrm{p}(2 \times 2)$ silicide is 5.0 $\mathrm{ML}$ and $\mathrm{Fe} / \mathrm{Si}(111)-7 \times 7$ is $7.5 \mathrm{ML}$. The $\mathrm{c}(8 \times 4)$ silicide effectively minimizes the MDL since it has a stable interface structure due to the high disintegration temperature. MDL on novel electric materials such as graphene [14], topological insulators [33] has not been well understood. Future works on the FM order and MDL of ultrathin Fe films on the interfaces will have important implications for both fundamental physics and the emerging spintronics technology.

\section{ACKNOWLEDGMENTS}

This work was supported by JSPS KAKENHI Grant Numbers JP25287077 and JP15H03677 and Advanced Graduate Program in Global Strategy for Green Asia (Kyushu University). 
[1] Y. Ufuktepe and M. Onellion, Solid State Commun. 76, 191 (1990).

[2] J. Alvarez, A. L. Vázquez de Parga, J. J. Hinarejos, J. de la Figuera, E. G. Michel, C. Ocal, and R. Miranda, Phys. Rev. B 47, 16048(R) (1993).

[3] M. Matsumoto, K. Sugie, T. Kawaguchi, K. Fukutani, and T. Okano, Jpn. J. Appl. Phys. 45, 2390 (2006).

[4] M. R. Scheinfein, K. E. Schmidt, K. R. Heim, and G. G. Hembree, Phys. Rev. Lett. 76, 1541 (1996).

[5] D. Berling, G. Gewinner, M. C. Hanf, K. Hricovini, S. Hong, B. Loegel, A. Mehdaoui, C. Pirri, M. H. Tuilier, and P. Wetzel, J. Magn. Magn. Mater. 191, 331 (1999).

[6] A. A. Alekseev, I. A. Kuyanov, and A. V. Zotov, Tech. Phys. 54, 1561 (2009).

[7] G. Garreau, S. Hajjar, J. L. Bubendorff, C. Pirri, D. Berling, A. Mehdaoui, R. Stephan, P. Wetzel, S. Zabrocki, G. Gewinner, S. Boukari, and E. Beaurepaire, Phys. Rev. B 71, 094430 (2005).

[8] K. Kataoka, K. Hattori, Y. Miyatake, and H. Daimon, Phys. Rev. B 74, 155406 (2006).

[9] P. Castrucci, R. Gunnella, R. Bernardini, M. De Crescenzi, L. Ferrari, C. Crotti, C. Comiccioli, C. Ottaviani, G. Gubbiotti, and G. Carlotti, Surf. Sci. 476, 43 (2001).

[10] M. Eddrief, Y. Wang, V. H. Etgens, D. H. Mosca, J. L. Maurice, J. M. George, A. Fert, and C. Bourgognon, Phys. Rev. B 63, 094428 (2001).

[11] M. V. Gomoyunova, D. E. Malygin, and I. I. Pronin, Phys. Solid State 50, 1579 (2008).

[12] K. Paredis, D. Smeets, and A. Vantomme, New J. Phys. 11, 093019 (2009).

[13] F. Zavaliche, W. Wulfhekel, H. Xu, and J. Kirschner, J. Appl. Phys. 88, 5289 (2000).

[14] W. Q. Liu, W. Y. Wang, J. J. Wang, F. Q. Wang, C. Lu, F. Jin, A. Zhang, Q. M. Zhang, G. van der Laan, Y. B. Xu, Q. X. Li, and R. Zhang, Sci. Rep. 5, 11911 (2015).

[15] Y. T. Cheng, Y. L. Chen, M. M. Karmarkar, and W. L. Meng, Appl. Phys. Lett. 59, 953 (1991).

[16] J. M. Gallego, J. M. Garcia, J. Alvarez, and R. Miranda, Phys. Rev. B 46, 13339 (1992).

[17] S. M. Rezende, J. A. S. Moura, F. M. de Aguiar, and W. H. Schreiner, J. Magn. Magn. Mater. 140-141, 663 (1995).

[18] Z. H. Nazir, C. K. Lo, and M. Hardiman, J. Magn. Magn.
Mater. 156, 435 (1996).

[19] M. Fanciulli, S. Degroote, G. Weyer, and G. Langouche, Surf. Sci. 377-379, 529 (1997).

[20] R. Kläsges, C. Carbone, W. Eberhardt, C. Pampuch, O. Rader, T. Kachel, and W. Gudat, Phys. Rev. B 56, 10801 (1997).

[21] S. Yaegashi, T. Kurihara, and K. Satoh, J. Appl. Phys. 81, 6303 (1997).

[22] S. Foss, C. Merton, R. Proksch, G. Skidmore, J. Schmidt, E. D. Dahlberg, T. Pokhil, and Y. T. Cheng, J. Magn. Magn. Mater. 190, 60 (1998).

[23] M. Cougo dos Santos, J. Geshev, J. E. Schmidt, S. R. Teixeira, and L. G. Pereira, Phys. Rev. B 61, 1311 (2000).

[24] P. Castrucci, R. Gunnella, R. Bernardini, P. Falcioni, and M. De Crescenzi, Phys. Rev. B 65, 235435 (2002); G. Gubbiotti, G. Carlotti, F. D'Orazio, F. Lucari, R. Gunnella, and M. De Crescenzi, Surf. Sci. 454-456, 891 (2000).

[25] S. M. Rezende, J. A. S. Moura, F. M. de Aguiar, and W. H. Schreiner, Phys. Rev. B 49, 15105 (1994).

[26] R. Naik, C. Kota, J. S. Payson, and G. L. Dunifer, Phys. Rev. B 48, 1008 (1993).

[27] Y. Shin, D. A. Tuan, Y. Hwang, T. V. Cuong, and S. Cho, J. Appl. Phys. 113, 17C306 (2013).

[28] A. N. Hattori, K. Hattori, K. Kodama, N. Hosoito, and H. Daimon, Appl. Phys. Lett. 91, 201916 (2007).

[29] K. Eguchi, Y. Takagi, T. Nakagawa, and T. Yokoyama, Phys. Rev. B 85, 174415 (2012).

[30] M. Krause, F. Blobner, L. Hammer, K. Heinz, and U. Starke, Phys. Rev. B 68, 125306 (2003).

[31] S. Hajjar, G. Garreau, S. Pelletier, D. Bolmont, and C. Pirri, Phys. Rev. B 68, 033302 (2003).

[32] Z. Q. Qiu and S. D. Bader, Rev. Sci. Instrum. 71, 1243 (2000).

[33] J. Li, Z. Y. Wang, A. Tan, P. A. Glans, E. Arenholz, C. Hwang, J. Shi, and Z. Q. Qiu, Phys. Rev. B 86, 054430 (2012).

[34] A. N. Hattori, K. Hattori, K. Kataoka, E. Takematsu, A. Ishii, F. Komori, and H. Daimon, J. Magn. Magn. Mater 363, 158 (2014).

[35] Y. Takagi, A. Nishimura, A. Nagashima, and J. Yoshino, Surf. Sci. 514, 167 (2002). 